\title{
Recent Advancements in the Infrared Flow Visualization System for the NASA Ames Unitary Plan Wind Tunnels
}

\author{
Theodore J. Garbeff II ${ }^{*}$, Jennifer K. Baerny ${ }^{\dagger}$ \\ NASA Ames Research Center, Moffett Field, Ca, 94035
}

The following details recent efforts undertaken at the NASA Ames Unitary Plan wind tunnels to design and deploy an advanced, production-level infrared (IR) flow visualization data system. Highly sensitive IR cameras, coupled with in-line image processing, have enabled the visualization of wind tunnel model surface flow features as they develop in real-time. Boundary layer transition, shock impingement, junction flow, vortex dynamics, and buffet are routinely observed in both transonic and supersonic flow regimes all without the need of dedicated ramps in test section total temperature. Successful measurements have been performed on wingbody sting mounted test articles, semi-span floor mounted aircraft models, and sting mounted launch vehicle configurations. The unique requirements of imaging in production wind tunnel testing has led to advancements in the deployment of advanced IR cameras in a harsh test environment, robust data acquisition storage and workflow, real-time image processing algorithms, and evaluation of optimal surface treatments. The addition of a multi-camera IR flow visualization data system to the Ames UPWT has demonstrated itself to be a valuable analyses tool in the study of new and old aircraft/launch vehicle aerodynamics and has provided new insight for the evaluation of computational techniques.

\section{Introduction}

Surface temperature gradients on wind tunnel test models are influenced by flow phenomenon.

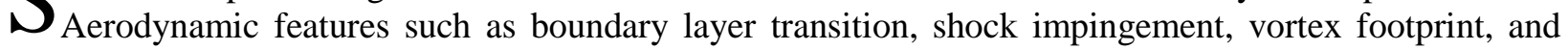
buffet all create temperature gradients on a test article. Modern research-grade infrared (IR) cameras boast both high-sensitivity $(<25 \mathrm{mK})$ and high-definition (megapixel) with an assortment of optics in a robust package. Coupling these cameras with server-class acquisition hardware and inline image processing has

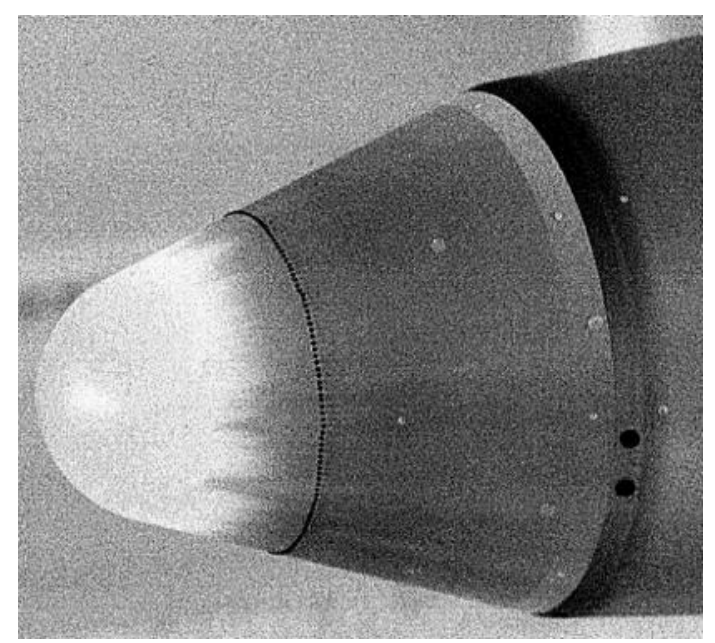

Figure 1: Boundary Layer Transition resulted in an imaging system capable of depicting an array of surface flow features as they develop in real-time without the need to impose temperature gradients by varying test section total temperature. This has led to a truly passive technique that poses no impact to the productivity of the wind tunnel test. Successful measurements have been performed at the 11 by 11 foot transonic test section of the NASA Ames Unitary Plan Wind Tunnel (UPWT) on wingbody sting mounted test articles, semi-span floor mounted aircraft models, and sting mounted launch vehicle configurations. Figure 1 demonstrates an example image of boundary layer transition occurring at the sphere-cone junction ahead of a boundary layer trip line on the nose of a generic launch vehicle. The drastic change in grayscale from light to dark represents a subsequent increase in heat transfer due to the transition to a turbulent boundary layer. The real-

\footnotetext{
* Instrumentation Engineer, Wind Tunnel Systems Branch, MS 227, Member AIAA

${ }^{\dagger}$ Instrumentation Engineer, Jacobs Technology Inc.
}

American Institute of Aeronautics and Astronautics 
time evaluation of boundary layer trip effectiveness has made this technique integral to the evaluation of new model geometries. Figure 3 details a partial view of the upper wing surface of an advanced ultra-green aircraft concept. A sharp greyscale gradient traveling span-wise from wing-root to wing-tip indicates the upper surface shock location. The dark band immediately following the discontinuity is indicative of shock induced separation. This particular configuration lacked boundary layer trips in order to study natural transition. Surface imperfections with accompanying turbulent wedges are apparent in the center of the image. As the local flow within these wedges is turbulent, the difference in severity between laminar and turbulent separation across a shock is demonstrated as the dark band of shock induced separation is visible where the flow is laminar but nonexistent where the flow is turbulent within the wedge. This technique provides a powerful tool to render complex flow fields across a wide range of model types. While valuable for its ability to assess boundary layer trip strategies a whole range of additional flow features and their interactions may be made visible.

The standard IR imaging configuration at Ames UPWT consists of four FLIR SC8200 series mid-wave (3-5 um), megapixel, research grade IR cameras with

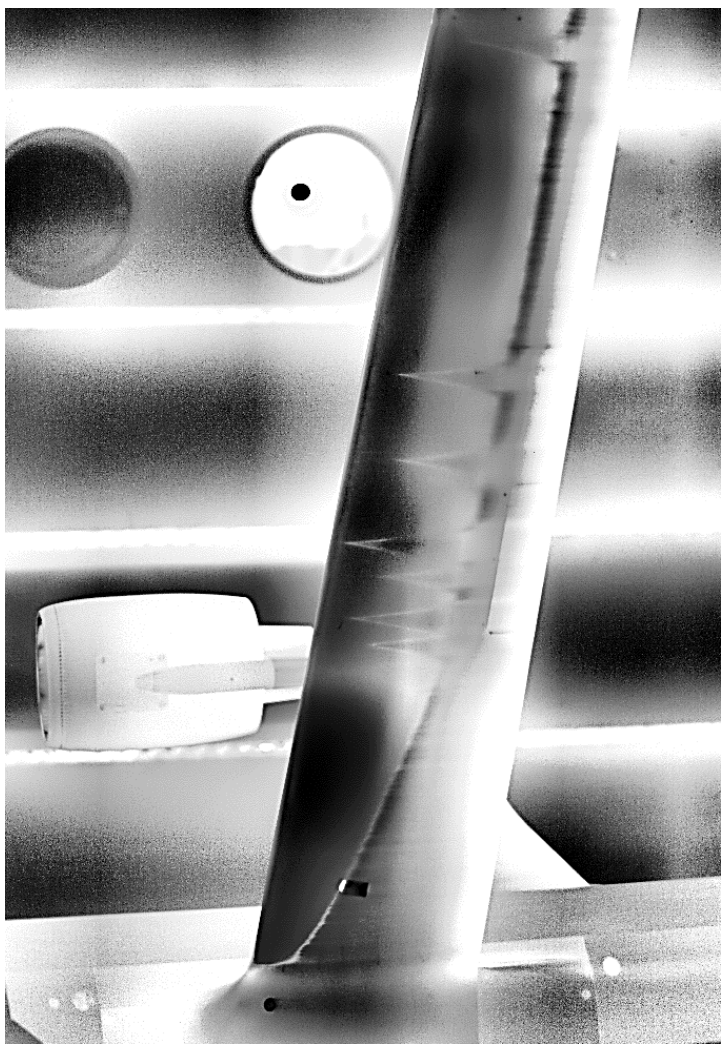

Figure 3: Shock Impingement / Separation supporting optics, hardware, and LabVIEW based data systems. Network published shared variables provide a link between each local IR data system and the wind tunnel standard data system, a detailed overview of the wind tunnel data system architecture is depicted in Figure 2. The raw data product is recorded as 14bit integer arrays while a particular flow feature may be evident as a gradient on the order of tens of counts. This makes the processing of the image data critical to the success of this technique. Additionally some amount of image processing must be performed in-line with image acquisition as it is a

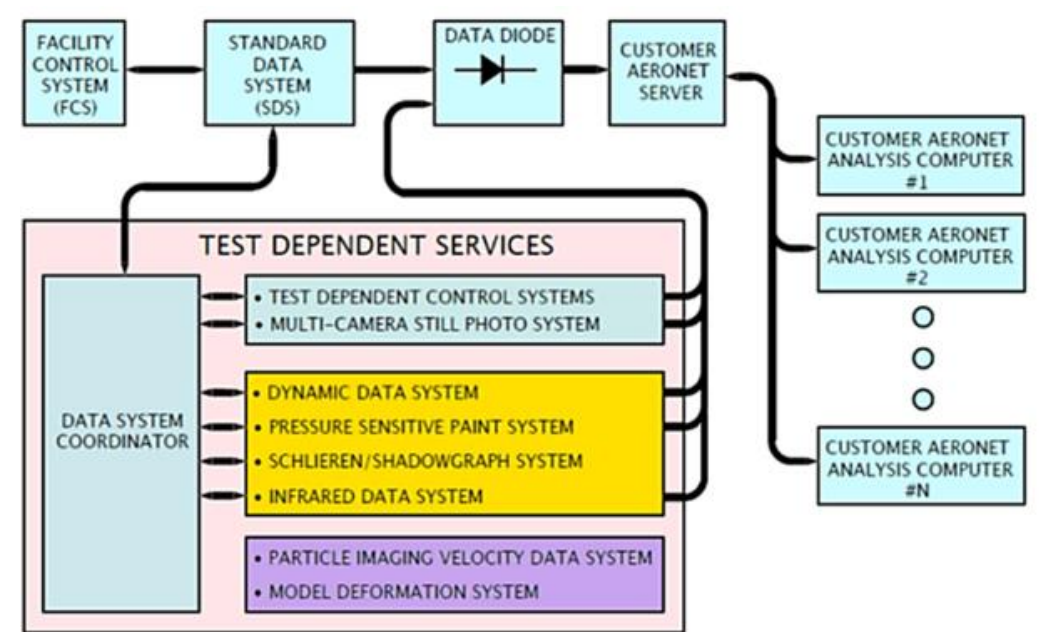

SERVICE SUPPLIED BY

WIND TUNNEL OFS

W WIND TUNNEL OFS AND AEROFHISICS ERANCH 口 AEROFHSICS BRANCH

Figure 2: Wind Tunnel Data Systems Architecture goal of this system to have its data products available real-time. Several strategies were explored and ultimately an in-line, contrast limited adaptive histogram equalization (CLAHE) image processing routine has been implemented. CLAHE is a contrast enhancement method originally developed for use in medical imaging ${ }^{1}$ and aircraft cockpit displays ${ }^{2}$. It is particularly well suited for this application as it is an automatic operation that maximizes all contrast available within the image. This coupled with an image overlay of current test conditions provides a realtime data product that is distributed throughout the test 
area to interested parties. An in-depth discussion of this image processing strategy as well as others shall be given.

Acquisition of all four cameras is triggered automatically by the wind tunnel data system via a data systems coordinator in parallel with all other test measurements. During acquisition, raw image data is streamed to disk along with current test conditions in binary format and organized based upon the test conditions at acquisition. Upon completion of acquisition, postprocessing is enqueued and the raw data products are rendered based upon the tagged image file format (TIFF) and audio video interleave (AVI) format, stored locally on a RAID1 array, with a subset of data products securely transferred to the researcher via a data diode.

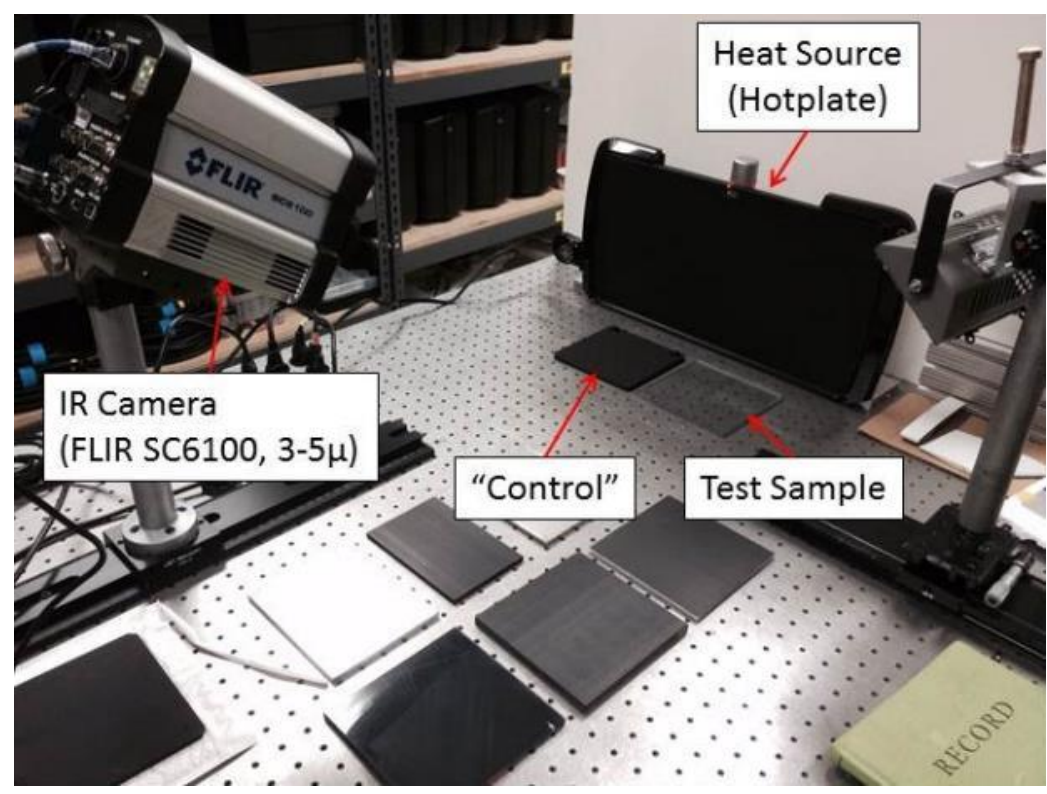

Figure 4: Surface Treatment Characterization

The quality of results for this technique depends greatly upon the surface treatment of the test article being imaged. A surface treatment that is high in infrared emissivity and low in thermal conductivity is critical in order to establish a high signal to noise ratio in the image data. A limited survey of various coatings has been performed in order to optimize these properties while minimizing any potential

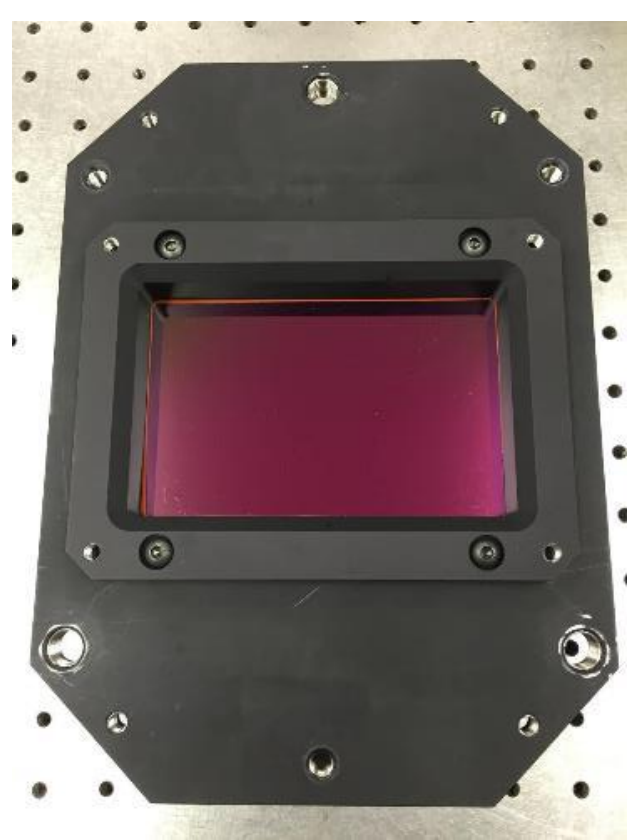

Figure 5: Modular Germanium IR

\section{Figure 5: Modular Germanium IR
Window} aerodynamic impact on the test article. The details of a small laboratory setup (Figure 4) to characterize these parameters will be given as well as results for various surface treatments.

Incorporating a multi-camera IR flow visualization data system into a production wind tunnel test environment has produced a unique set of challenges. Care must be taken to simulate multicamera fields of view, plan camera locations, and deploy dedicated IR transparent (Germanium and Sapphire) viewing ports (Figure 5). To that end, an overview of typical IR measurement test planning techniques will be given. The addition

- of a multi-camera IR flow visualization data system to the Ames - UPWT has demonstrated itself to be a valuable analyses tool in the study of new and old aircraft/launch vehicle aerodynamics and has provided new insight for the evaluation of computational techniques. Deploying sensitive IR cameras coupled with realtime image processing and robust data acquisition has revealed a surprisingly dynamic and informative capability. A tool to aid the researcher and designer in developing the next generation of aircraft and spacecraft. 


\section{References}

${ }^{1}$ S. M. Pizer, E. P. Amburn, J. D. Austin, et al.: Adaptive Histogram Equalization and Its Variations. Computer Vision, Graphics, and Image Processing 39 (1987) 355-368.

${ }^{2}$ D. J. Ketcham, R. W. Lowe \& J. W. Weber: Image enhancement techniques for cockpit displays. Tech. rep., Hughes Aircraft. 1974.

American Institute of Aeronautics and Astronautics 